\title{
Study on the fiber fouling in drying exhaust heat utilization of wood industry
}

\author{
Yadi Pan *, Shuya Shan, Yao Wei, Feng Ji, Jinping Weng, Yulan Tian and Jin Qian \\ Nanjing Forestry University;
}

\begin{abstract}
Based on the viscoelastic and creep deformation properties, a new deposition mechanism model for slender wood fiber particles in wast heat utilization is proposed in this paper. And the equivalent sphere method is used to describe the particle feature size in the model. With the proposed deposition model of flexible slender particles, the critical criteria are obtained. The influence of particle size, aspect ratio and damping factor on particle deposition has been investigated. The results indicate that particle deposition increases with the particle size decrease, aspect ratio and damping factor increase. According to the present deposition model, a coupling simulation with FLUENT and EDEM method was carried out for the flow field of fiber drying tail gas in heat piping exchanger, which indicated that particle deposition mainly occurred at the central windward area of fin due to the direction changer and the magnitude decrease of collision velocity between fiber particles and wall. Experiment of heat recovery of drying tail gas revealed that using the $\mathrm{H}$ fin tubes instead of rectangular fin tubes can greatly relieve the deposition of wood fiber particles, which provided a useful way to save energy in wood industries.
\end{abstract}

\section{Introduction}

In the wood fabrication industry, there are large amount of fiber drying exhaust, where the temperature is usually about $80{ }^{\circ} \mathrm{C}$. An annual output of exhaust gas could be produced up to about $250,000 \mathrm{~m}^{3} / \mathrm{h}$ in a $100,000 \mathrm{~m}^{3}$ middle density fiberboard production line, which can get about $180,000 \mathrm{~kW}$ waste heat if they are cooling to $40^{\circ} \mathrm{C}$. However, the deposition of fiber particles in the heat exchanger has increased the cost of equipment operation, and decreased the efficiency of heat recovery. Therefore, the mechanism of fiber deposition is one of hot topics in the research of energy saving and emission reduction in the current wood industry.

There have been relevant research of the collision deposition mechanism for elastic particles on the rigid wall and such mechanism was used as guideline in practical applications. However, as a kind of polymer, the creep deformation of viscoelastic wood fiber would occur under the external force load. Furthermore, fiber particle is slender, whose aspect ratio is greater than 1. So far, the deposition mechanism of such flexible slender particles has rarely been reported. Feng et al. [1] studied the deposition of elastoplastic particles on the wall, and analyzed the influence of adhesion between the particles and the wall on the deposition of elastic plastic particles under different velocity. It concluded that the influence of the interface adhesion is more important than that of the plastic deformation at the low speed. Marshall et al. [2] analyzed the behavior of the particles after its collision with the wall in suspension flow, such as rebound, deposition and tumbling. And it pointed out that the particle will roll instead of sliding after its collision with the wall because of the impact of air flow. The recovery coefficient of particle-wall collision was studied by Troiano and co-workers [3], which including coke sawdust and wood chips. In literature [4], the fluidization and agglomeration of the slender particles that was divided into a plurality of rigid segments by hinges were researched. Lin et al. [5] put forward the control equations of particle sedimentation in static water based on the cylindrical particle oscillation frequency $\omega$, which considered the influences of the oscillation, the torque and inter force of particles. Based on the lattice Boltzmann method, Lin et al. [6-8] studied the influences of the aspect ratio, wall constraint and particles interaction on the settlement of slender particles, which reveals the orientation and motion characteristic of particles.

Aewald algorithm, based on slender body theory, was used in the research of Saintillan et al.[9] to simulate the settlement of non-Brown rigid slender particles under the action of gravity and a Reynolds number of 0 . The numerical results showed that the fibers with different sizes were aggregating continuously during the settlement process. And the particles would separate from the cluster when the cluster reached a certain size, and then aggregated with other particles to another clusters. Based on JKR theory and adhesive elastic contacts, Marshallin et 
al. [10] studied three-dimensional simulations of micro-particle deposition and aggregation on an individual fiber of an array using the discrete element method (DEM). The simulation results showed that the fluid viscosity and velocity would affect the particle capture, and the particle deposition occurred in a narrow area near the front fiber line due to the effect of high shear force and particle shadow on the surface of fiber. Helzel et al. [11] proposed columnar Brown particles settlement kinetic model of low Reynolds number flow in dilute suspension, using the multi-scale dynamics model to describe the suspended particles, which could be used to describe the cluster formation for high concentration of particle flow. All of these studies provide valuable

\section{Deposition model}

Some assumptions were made to simplify the analysis model: (1) the deformation of the metal wall was ignored due to the deformation of viscoelastic creep of wood fibers is much bigger than that of the metal wall; (2) The mass and radius of wall was considered infinite with the comparison of the fine dust particles; (3) The collision of particle clusters with the wall surface was not considered as the particles are not easily collided with each other in the low dust concentration. We only analyzed the collision the single particle and wall.

\subsection{The force analysis of particles in the flow field}

The dust concentration distributes in the range of $120 \sim 180 \mathrm{mg} / \mathrm{m}^{3}$ in the fiber dry exhaust heat utilization, in which the particle concentration is relatively low. Stokes model was taken in the study the collision between particles and walls. Particles of 80 160 mesh size were analyzed in this paper. The screening statistical analysis shows that the aspect ratio is less than 4 . Select volume equivalent sphere model was used for deposition mechanism of particle analysis, and we used the volume shape factor to modify this model. Volume equivalent sphere diameter fiber particle $\mathrm{d}_{\mathrm{V}}$ is shown by the equation (1).

$$
d_{V}=\sqrt[3]{6 V / \pi}
$$

Where $\mathrm{V}$ in (1) is the actual volume of a particle. And the particle volume shape coefficient $\varphi_{\mathrm{V}}$ is shown as following:

$$
\varphi_{V}=V / \mathrm{d}^{3}=\pi d_{V}^{3} /\left(6 d^{3}\right)
$$

Where $d$ in (2) is the actual transverse particle size of particle.

When fiber dust moved and collides with the wall in a flow field, it suffers multitudinous forces including the collision normal impact force $F_{\text {in }}$, gravity $F_{g}$, buoyancy references for the analysis on particle deposition mechanism of wood fiber, but there are still a lot of work needs to be further researched on the deposition mechanism of slender particles considering its viscoelastic properties due to the unique stress relaxation and energy consumption characteristics.

Due to the variety of the components, size and shape of the wood fiber particles emitted by wood enterprise, with a large number of water vapor. The process of fiber deposition is affected by many factors and deposition mechanism is really complex. To simplify the analysis, this paper would do some exploratory research on the deposition mechanism of wood particles.

$F_{b}$, lift $F_{l v}$, adhesion $F_{a d h}$, surface normal force $F_{t n}$, tangential impact force $F_{i t}$, drag force $F_{d}$, friction force $F_{f}$, which are shown in Figure 1.
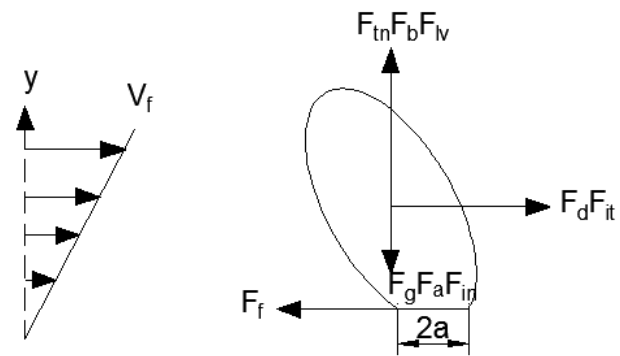

Fig. 1. The force analysis of the target particles.

The particle size of 95 200 $\mu \mathrm{m}$ (mesh size: $80-160$ ) are selected in this study, whose equivalent spherical diameter ranges from 140 to $360 \mu \mathrm{m}$ by formula (1). These particles belong to micron-sized powder particles, their magnitude of the contact surface adhesion force is 1000 times of gravity [12-13], so the influence of gravity of particles $F_{g}$ in deposition is very small, which can be neglected. The air density is much smaller than the particles', so the buoyancy is also negligible. Lift Flv can be expressed as: $\mathrm{F}_{\mathrm{lv}}=1.625 \rho_{\mathrm{f}} \mathrm{d}_{\mathrm{p}}^{2} \sqrt{\mathrm{v}_{\mathrm{f}}} \mathrm{u}_{\mathrm{r}} \sqrt{\partial \mathrm{u} / \partial \mathrm{y}}$, it is related to the fluid viscosity $v_{f}$, the velocity gradient along the direction of the fluid particles, and the relative speed $u_{r}$, eta [14-15], and $V_{f}$ is the air flow rate. The lift of particles is usually ignored due to small air velocity gradient $u$ along the $y$ direction for such size particles. By analysis of force on the particle, the force balances the equation of the particles in the vertical direction can be expressed as equation (3):

$$
F_{t n}-F_{\text {in }}-F_{\text {adh }}=0,
$$

In the same way, the forces balance, i.e. the drag force $F_{d}$, tangent impact force $F_{i t}$ and friction force $F_{f}$, acting on the particles in the horizontal direction, are shown by equation (4).

$$
F_{d}+F_{i t}=F_{f}
$$

Where flag fore is: $F_{d}=C_{D} \pi \rho_{f} u_{r} d_{p}^{2} / 8, C D$ is the drag coefficient, and friction force is $F_{f}=f^{*} F_{n}$, in which $F_{n}=-F_{t}$, and $f^{*}$ is effective friction coefficient. 


\subsection{Normal deposition model}

High polymer has typical characteristics of the viscoelastic. We proposed a viscoelastic damping contact mechanics model for wood fiber based on the lag contact mechanics model called Hertz-Flores [16]. And the normal contact force of the particle when it collides with wall can be expressed as:

$$
F_{\text {in }}=K_{\text {in }} \delta+\chi \delta V_{\text {in }}
$$

The first item in the right side of the equation is elastic force, $K_{i n}$ is the stiffness coefficient, $K_{i n}=\frac{4}{3} E^{*}\left(R^{*}\right)^{\frac{1}{2}}$, where $\mathrm{R} *$ is the effective radius in collision system, $1 / R^{*}=1 / r_{1}+1 / r_{2}$, Where $r_{1}$ and $r_{2}$ represents particle and peace wall radius respectively, and $\mathrm{E}^{*}$ is effective elastic modulus, $1 / \mathrm{E}^{*}=\left(1-\mathrm{v}_{1}^{2}\right) / \mathrm{E}_{1}+$ $\left(1-v_{2}^{2}\right) / E_{2}$, where $v_{1}$ and $v_{2}$ are particles and wall Poisson ratio respectively, $\mathrm{E}_{2}$ is wall elastic modulus, $\mathrm{E}_{1}$ is relaxation modulus for viscoelastic fiber particles, which could be describe by complex form: $\mathrm{E}_{1}=\mathrm{E}^{\prime}+\mathrm{iE}^{\prime \prime}$, where $E^{\prime}$ is storage modulus, $E^{\prime \prime}$ is loss modulus. The second item is the viscous dissipation force, where $\chi$ is hysteresis damping factor. It is related to the structure of the material, viscosity, water content, temperature and so on, " $\delta$ " is the normal contact displacement and $V_{\text {in }}$ is the normal relative velocity.

According to JKR theory and Hertz-Flores [16] theory, when wood fiber particles collide with the wall, the energy conservation equation is shown as equation (6) when the normal compression distance of particle collision reaches the maximum value.

$$
Q_{\text {in }}+Q_{a d h}=Q_{e}+Q_{n h}
$$

Where Qin is the impact kinetic energy before the collision of particles, $Q_{\mathrm{adh}}$ is the adhesion energy between the particles and the wall during the approaching process, $\mathrm{Q}_{\mathrm{adh}}=2 \pi \mathrm{a}_{0}^{2} \omega_{1}$, in which $\mathrm{a} 0$ is the initial radius of the contact surface between the contact surface only by the adhesion force $F_{a d h}, F_{a d h}=3 \pi R^{*} \omega_{1}$, where $\omega_{1}$ is the surface energy of the two collision during the approaching process, $\mathrm{Q}_{\mathrm{e}}$ is elastic storage of wood fiber particles in the energy dissipation after the collision, $\mathrm{Q}_{\mathrm{e}}=\int_{0}^{\delta_{\max }} \mathrm{F}_{\mathrm{n}} \mathrm{d} \delta=\frac{2}{5} \mathrm{~K} \delta_{\max }^{5 / 2}$; and $\mathrm{Q}_{\mathrm{nh}}$ is the dissipated energy which means irreversible work of wood fiber particles to overcome the internal friction and the molecular chain segment movement, $\mathrm{Q}_{\mathrm{nh}}=\chi \mathrm{E}^{*}\left(\mathrm{R}^{*}\right)^{0.5} \delta_{\text {max }}^{2.5} / 2$, including high elastic deformation and viscous friction flow friction.

After collision, energy dissipation will become heat existing in the collision system, while the elastic energy would be stored in two ways, one of which is used to overcome the adhesion between the collision bodies and restore the deformation, the other part is transformed into the particle kinetic energy which makes the particle rebound off the wall in the velocity of $\mathrm{V}_{\mathrm{rn}}$. The process meets the energy conservation following equation (7).

$$
\frac{1}{2} m^{*} V_{r n}^{2}=Q_{e}-Q_{a d h^{\prime}}^{\prime}
$$

Where $\mathrm{m}^{*}$ is the effective collision mass: $1 / \mathrm{m}^{*}=$ $1 / \mathrm{m}_{1}+1 / \mathrm{m}_{2} ; \mathrm{Q}_{\mathrm{adh}}^{\prime}$ is the adhesion energy that particles must be overcome during the separating process, $\mathrm{Q}_{\mathrm{adh}}^{\prime}=$ $2 \pi \mathrm{a}^{2} \omega_{2}$, where $\omega_{2}$ is the particle interface energy in the separating process. Literature [1] studied that $\omega_{-} 2$ in the separating process is usually much bigger than $\omega_{1}$ in the approaching process, especially for viscoelastic particles, the number between the them is relatively large; and a is equivalent radius of contact surface area after the collision.

For meet the requirement of equation (7), that is, the elastic energy storage of particles is equal to the adhesion energy that particles need to overcome when they leave the deposition surface, the normal velocity components of particles impinging on the wall are defined as the critical velocity $\mathrm{V}_{\mathrm{in} \_ \text {cri }}$, described by equation (8) as following:

$$
\begin{gathered}
V_{\text {in_cri }}= \\
f\left(\varphi_{V}, R^{*}, m^{*}, E^{*}, \chi, \omega_{1}, \omega_{2}\right),
\end{gathered}
$$

\subsection{Tangential deposition model}

When the particle tangential impulse is larger than the rolling friction resistance, which satisfies equation (9), the particles will roll down and get out of the wall. Otherwise, the particles will deposit.

$$
F_{t}=F_{d}+F_{i t} \geq f^{*}\left(F_{i n}+F_{a d h}\right),
$$

Where $F_{i t}$ is the tangential component of particle contact force. According to the literature [17]: $F_{i t}=$ $\min \left(\mathrm{f}^{*} \mathrm{~F}_{\mathrm{in}}, \mathrm{K}_{\mathrm{t}} \mathrm{V}_{\mathrm{it}} \Delta \mathrm{t}\right)$, where $\Delta \mathrm{t}$ is the contact time. And according to the article [18], the recovery of tangential motion after the particles collision on the premise that action time of the tangential impact force is at least equal to the impact time of the normal's. In this paper, the correction coefficient a is used to multiply the normal contact time: $\Delta \mathrm{t}=1.47 \mathrm{a}\left(5 \mathrm{~m}^{*} / 4 \mathrm{n}\right)^{0.4} \mathrm{~V}_{\text {in }}^{-0.2}, \mathrm{n}=$ $4 \mathrm{E}^{*} \sqrt{\mathrm{R}^{*}} / 3$, and the incident angle $\theta$ is defined: $\tan \theta=$ $\mathrm{V}_{\mathrm{it}} / \mathrm{V}_{\mathrm{in}}$. According to the definition of drag force, the air drag force on particle is very small, which can be ignored. While due to such small size of fiber particles, the effect of adhesion force can also be ignored comparing to the inertial impact force in the collision process.

As a result, the equation (9) can be transformed into a function of the impact angle. The minimum impact angle of the collision particles rolling away from the wall is defined as the critical impact angle: $\theta_{\text {it_cri }}$, and equation (10) could be gotten:

$$
\theta_{i t_{-} c r i}=f\left(f^{*}, \chi, K_{i t}, K_{i n}\right),
$$

In summary, the criteria for collision deposition of wood fiber particles and walls could be described as follows:

(1) when $\theta>\theta_{\text {it_cri }}$, the particles will continue to move along with the fluids and will not be deposited on the wall;

(2) when $\theta<\theta_{\text {it_cri }}$ and $V_{\text {in }} \geq V_{\text {in_cri }}$, the particle will rebound away from the wall; 
(3) when $\theta<\theta_{\text {it_cri }}$ and $V_{\text {in }}<V_{\text {in_cri }}$, the particles will deposit on the wall.

\section{Analysis of influencing factors}

\section{1. predictive parameters of model}

For analysis the influence of some factors on slender viscoelastic particle deposition, such as particles size, aspect ratio, damping factor and so on, the critical criteria of a case collision between wood fiber and wall is predicted based on the proposed model, with mesh size between 80 to 160 . And the calculation parameters of the wood fiber and wall are shown in Table 1.

Table 1. Calculation parameters of collision between particles and wall.

\begin{tabular}{|c|c|c|}
\hline \multicolumn{2}{|c|}{ element } & value \\
\hline \multirow{4}{*}{ wall } & $\begin{array}{c}\text { elasticity model } \\
\text { E2/(Mpa) }\end{array}$ & 230 \\
\cline { 2 - 3 } & $\begin{array}{c}\text { Poisson ratio } \\
\mathrm{v}_{2}\end{array}$ & 0.3 \\
\cline { 2 - 3 } & $\begin{array}{c}\text { Effective } \\
\text { friction } \\
\mathrm{f}^{*}\end{array}$ & 0.1 \\
\hline \multirow{5}{*}{$\begin{array}{c}\text { Wood fiber } \\
\text { particles }\end{array}$} & $\begin{array}{c}\text { Relaxation } \\
\text { model } \\
\text { E1/(Mpa) }\end{array}$ & 37.7 \\
\cline { 2 - 3 } & $\begin{array}{c}\text { Poisson ratio } \\
\mathrm{v}_{1}\end{array}$ & 0.4 \\
\cline { 2 - 3 } & $\begin{array}{c}\text { Density } \\
\mathrm{P} /(\mathrm{kg} \cdot \mathrm{m}-3)\end{array}$ & 500 \\
\hline
\end{tabular}

\section{2. influence factors}

Based on the model, fiber particles whose damping factor is 0.1 , the particle sizes of 100,150 and $200 \mu \mathrm{m}$ are selected to analyze the influence of the aspect ratio on the critical speed. The result is shown in Figure 2.

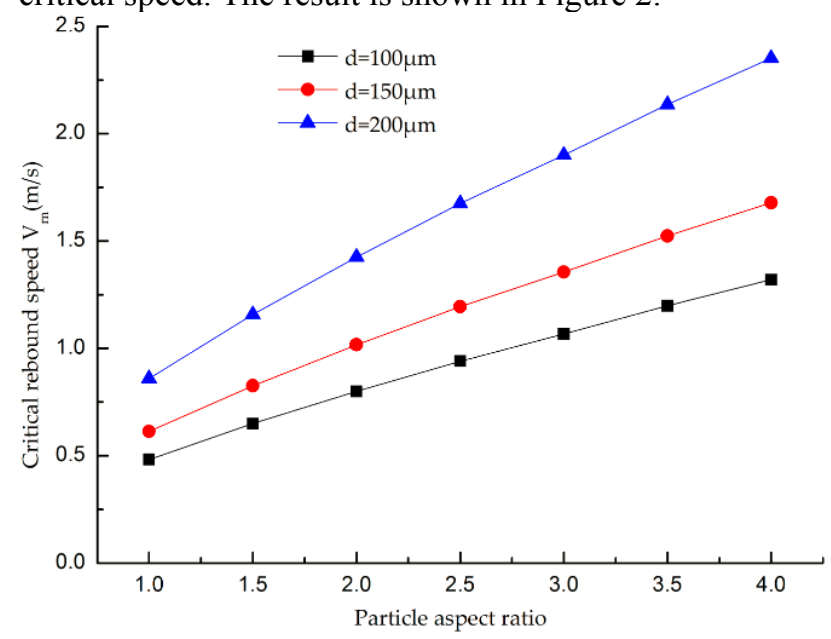

Fig. 2. Influence of aspect ratio on critical rebound velocity.

Theoretically, the large aspect ratio means particles are slenderer, and the viscosity would increase. With the same collision force, the dissipated energy is bigger, the adhesion energy needed to overcome during the separating progress from the wall surface is greater, which means the initial impact kinetic energy needed for critical rebound would be greater. The prediction results by the model shown in Figure 2 also showed that the critical rebound speed increase with the particle aspect ratio increases, which is consistent with the theoretical analysis.

The damping factor of wood fiber is different with the materials, structures and moisture content. According to the model, the influence of damping factor on critical speed was analyzed at a ratio of 2 with different particles diameter, and the result is shown in Figure 3.

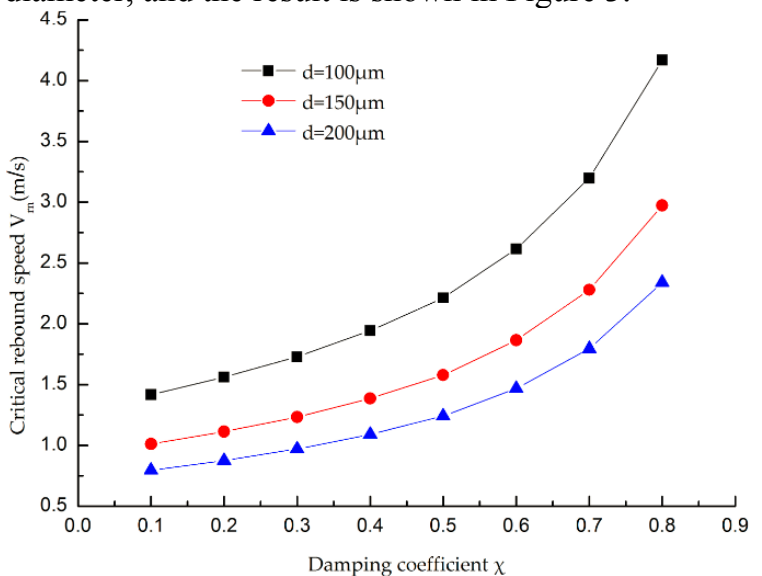

Fig. 3. The influence of damping factor on the critical rebound velocity.

Based on the viscoelastic theory proposed by Flores [16], when the damping coefficient of particles increases, the energy consumption in the process of collision is bigger, the elastic energy stored is smaller, and the required critical velocity of particle collision rebound would be bigger. the critical velocity in Figure 3 increases with damping coefficient, under the same particle size, which conforms to the theoretical analysis.

What's more, under the same aspect ratio and damping coefficient, the larger particle size, the greater particle mass and impact energy with the same impact velocity, which lead to the bigger the elastic energy and be easier for particles to rebound, namely, critical rebound speed is smaller. The model shows that the critical velocity is inversely proportional to the 1.2 th power of particle size, and the critical velocity decrease with the increase of particle size as shown in Figure 2 and 3, which consistent with the theoretical analysis. As for the particles with aspect ratio bigger 4, we need further study for those more flexible and curl morphology.

\section{Experiment and simulation analysis}

\section{1. simulation analysis}

A coupling simulation method with the FLUENT and EDEM software was used to simulate the movement of dry exhaust gas in the field of heat piping exchanger. The saw dust particle with the aspect ratio of $2,3,4$, and size $0.15 \mathrm{~mm}$ are used in the simulation. Through the axial 


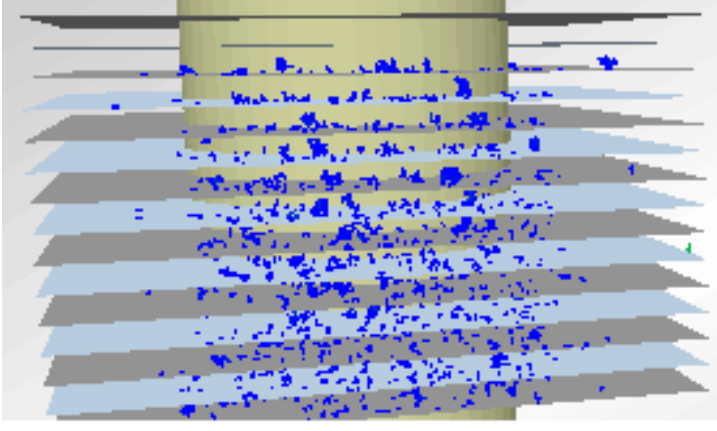

(a) view in the front of the tube

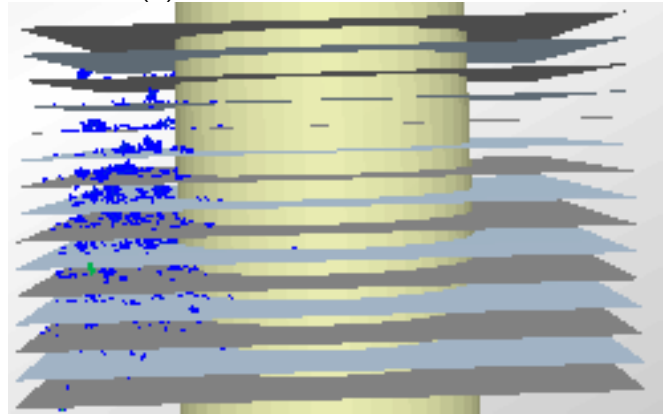

(b) view at the side of tube

Fig. 5. Simulation results of fibrous particle deposition.

superposition of the based particle, the slender geometry of the particle can be created with EDEM software, which surface smoothness can be adjusted by the superposition displacement and particle size. Figure 4 shows the simulation particle with size $0.15 \mathrm{~mm}$ and aspect 4 in EDEM.

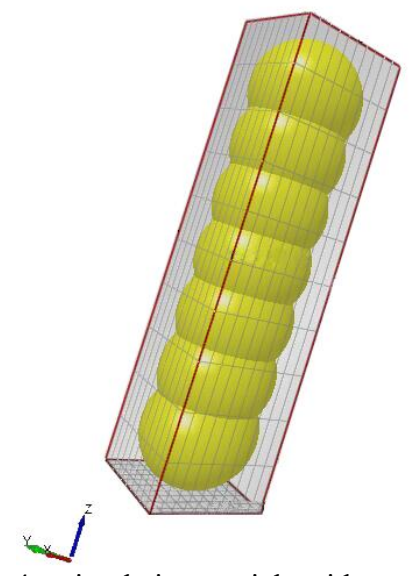

Fig. 4. simulation particle with aspect ratio of 4

According the slender saw particles, hysteretic damping factor of $0.1 \mathrm{~J} / \mathrm{m} 2$ is set up for measure the viscoelastic energy consumption in simulation. And the standard $\mathrm{k}-\varepsilon$ model was used to simulate the flow of fluid. Based on the present slender particulate deposition model, a User-defined function is embed in FLUENT software, which can obtain the impact velocity $\mathrm{V}$ and impact angle $\theta$ of particles near wall, as the flowing:

DEFINE_DPM_EROSION(dpm_accr,p,pt,f,normal ,alpha,Vmag,Mdot $) \backslash$

$$
\{\ldots . .
$$

$\mathrm{Vn}=\mathrm{Vmag} * \sin \left(\right.$ alpha); $\quad / *_{\text {obtain }}$ the normal velocity of impact particle*/
If (alpha>cita_cir) of the particle*/

return;

if $(\mathrm{V} n>\mathrm{V}$ in_cir)

velocity of impact particle*/

return;

else

F_UDMI $(\mathrm{f}, \mathrm{t}, 0)+=\mathrm{P} \_\mathrm{MASS}(\mathrm{P}) ; \quad / *$ storage the mass of deposition particles*/

F_UDMI $(f, t, 1)=$ num_in_data $+1 ; \quad / *$ storage the number of deposition particles*/

\section{-....}

\}

When the dry tail gas flow from to the heat piping, its flow characteristics changer greatly. When the particle flow to the central windward area, the velocity reduced and the direction changed for the formation of boundary layer along the cylinder, while these changes mach smaller in windward side region for the boundary layer along the plate. And the impact angle and velocity of the collision between particles and wall would decrease for the change of flow direction in the field. If the impact angle is less than the critical the impact angle, and the velocity of particle is less than the critical rebound also, which means $\theta<\theta_{\text {it_cri }}$ and $V_{\text {in }}<V_{\text {in_cri }}$, the particles will be deposited on the wall. Fiber particles around the the central windward area of tubes would easily match deposition critical than those around the side region of tubes. Therefor, the amount of fiber particle deposition on the central windward area is greater than on the windward edge area, as shown in figure 5 from simulation.

\section{2. experience and analysis}

At present, heat piping exchanger are usually applied in the waste heat utilization device of fiber drying desiccation in Chinese wood industry. In this research, the gravity heat pipe is used for experiment. The experimental device is shown in Figure 6.

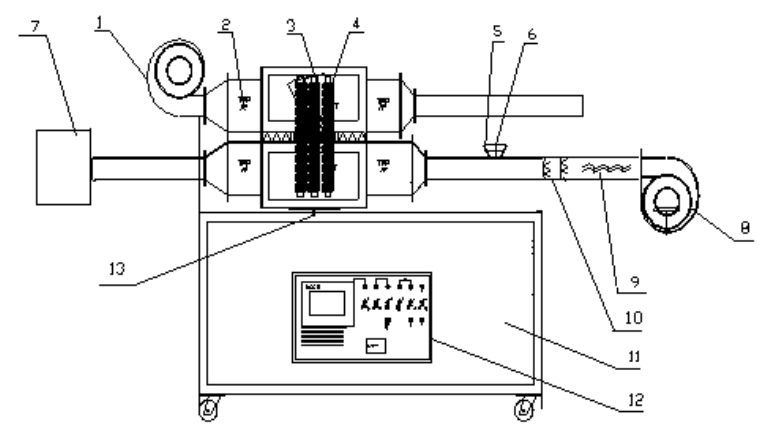

Fig. 6. Schematic diagram of an experimental device. 1. Cold fan; 2. Temperature, humidity, pressure test holes; 3 . heat pipe experiment section; 4. The rotary wheel; 5 . Flashboard; 6 . Ash adding port; 7. Dust elimination device; 8. hot end fan; 9. Electric heating device; 10. Humidifier; 11. Working table; 12. Control cabinet; 13. condensate discharge port

As the above simulation results shown, wood fiber particles mostly deposited at the central windward area of fin, both the rectangular fin tub and the $\mathrm{H}$ fin tub with a 
gap at the center were used to experiment. The size of wood fiber particles in the experiment is less than $0.2 \mathrm{~mm}$. When the inlet velocity of the dry tail gas $t$ is $3 \mathrm{~m} / \mathrm{s}$, the ash distribution on the surface of the fin surface of the heat pipe is shown in Figure 8(a) and (b) separately after 5 hours of operation.

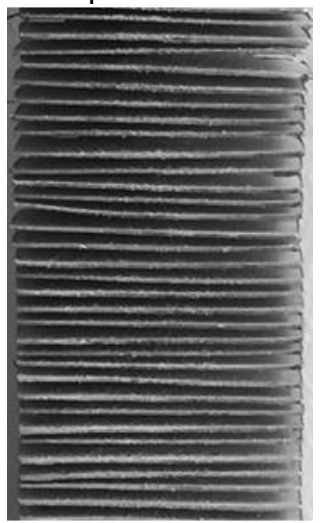

(a) deposition on rectangular fin

Fig. 7. Experimental results.

As shown in Figure 7(a), at the central windward area of rectangular fin, the particulate fouling is serious than the side of fin. While in Figure 7(b), as the central area of the fin is removed, the particle fouling on $\mathrm{H}$ fin is seldom, which can be guide for the waste heat recovery of middle density fiberboard production enterprises.

\section{Conclusions}

In this paper, the characteristics of wood fiber particles with size less than $0.2 \mathrm{~mm}$ under the external force load are studied. Combined with viscoelastic and creep deformation properties of wood fiber, a deposition mechanism model for slender fiber particles is proposed for the first time, and the equivalent sphere method is used to solve the model of blanket deposition mechanism of wood particles with aspect ratio less than 4 .

The predicted model indicates that the critical velocity of the wood fiber particles increases with the decrease of particle size, the increases of the aspect ratio and the damping factor, which are consistent with the theoretical analysis.

The coupling simulation with the FLUENT and EDEM method, using the present deposition mode, was carried out. The simulation results showed that the fouling deposition mainly occurred at the center fin of the front tube. And experiments on waste heat utilization of fiber drying tail gas were performed as well. Using the $\mathrm{H}$ fin tubes instead of rectangular fin tubes in heat exchanger can greatly relieve fouling deposition of wood fiber particles, which provides a useful way to solve the problem of the ash deposition. Although we do not have sufficient data to show the real profits we can get by our research in the real production lines in wood industries, we hope to contribute to successful development of the utilization of low temperature waste heat and efficiency improvement of heat exchanger for the energy-saving target.

\section{Acknowledgments}

This work was supported by the National Natural Science Foundation of China (Grant Number 51406088).

\section{References}

1. Xiqiao Feng. Huan Li, Hongping Zhao, Sholi-Wen Yu. Numerical simulations of the normal impact of adhesive microparticles with a rigid substance. POWDER TECHNOL, 189, 34(2009).

2. Marshall, J.S., Particles aggregation capture by walls in a particulate aerosol channel flow. J Aerosol Sci, 38, 333(2007).

3. Maurizio Troiano, Fabio Montagnaro, Piero Salatino, Roberto Solimene. Experimental characterization of particle-wall interaction relevant to entrained-flow gasification of biomass. FUEL, 19, 674(2017).

4. Fan Geng, Yingchao Wang, Yimin Li, Longji Yuan, Xinyong Wang, Min Liu, Zhulin Yuan. Numerical simulation on mixing dynamics of flexible filamentous particles in the transverse section of a rotary drum. PARTICUOLOGY, 11, 594(2013).

5. Jianzhong Lin, Weixiong Wang. Numercial simulation of the sedimentation of cylindrical particles. J Eng Thermophysrus Therm, 23, 631(2002).

6. Jianzhong Lin, Shi Xing, Zhenjiang You. Effects of the aspect ratio on the sedimentation of a fiber in Newtonian fluids. J Aerosol Sci, 34, 909(2003).

7. Jianzhong Lin, Changbin Wang, Xing Shi. Study on the effect of wall constraint on the settlement of columnar particles in Newton fluid. Chinese Journal of Applied Mechanics, 20, 2(2003).

8. Jianzhong Lin, Xueming Shao, Xing Shi, Zhaosheng Yu. Study on the interaction of sedimenting cylindrical particles in still fluid. Acta Mech Sinica, 20, 32(2004).

9. David Saintillan; Eric Darve; Eric S. G. Shaqfeh. A smooth particle-mesh Ewald algorithm for Stokes suspension simulations: The sedimentation of fibers. Phys Fluids, 17, 033301(2005).

10. Li S.-Q., Marshall J.S. Discrete element simulation of micro-particle deposition on a cylindrical fiber in an array. $\mathrm{J}$ Aerosol Sci, 38, 1031(2007).

11. Christiane Helzel, Athanasios E. Tzavaras. A kinetic model for the sedimentation of rod-like particles. Multiscale Model Sim, 15, 500(2017).

12. Abd-Elhady M.S., Rindt C.C.M, van Steenhoven A.A. Contact time of an incident particle hitting a $2 \mathrm{~d}$ bed of particles. Powder Technol, 191, 315(2009).

13. Anandarajah A. Multi-mechanism anisotropic model for granular materials. Int J Plasticity, 24, 804(2008).

14. Evgeny Rabinovich, Haim Kalman. Incipient motion of individual particles in horizontal particle-fluid systems: A. Experimental analysis. Powder Technol, 192, 318(2009).

15. Akhatov I. S., Hoey J. M., Swenson O.F., Schulz D.L. Aerosol focusing in micro-capillaries: Theory and experiment. J Aerosol Sci, 39, 691(2008).

16. Paulo Flores, Margarida Machado, Miguel T. Silva, Jorge M. Martins. On the continuous contact force models for soft 
materials in multibody dynamics. Multibody syst dyn, 25, 357(2011).

17. Schwager T., Becker V., Pöschel T. Coefficient of tangential restitution for viscoelastic spheres. The European Physical Journal E, 27, 107(2008).

18. Guangcheng Zhang, Huiming Tang, Xin Xiang. Characteristic parameters theoretical analysis of rockfall impact on ground. Chinese Journal of Rock Mechanics and Engineering, 31, 2839(2012). 